\title{
The site of brainstem lesions causing semicircular canal paresis: an MRI study
}

\author{
D A Francis, A M Bronstein, P Rudge, EPGH du Boulay
}

\begin{abstract}
Ten patients with canal paresis of central origin and ten patients with peripheral canal paresis were studied using MRI of the brainstem to identify lesions within the central vestibular pathways. In the central group, the magnitude of the canal paresis was generally lower than in the peripheral group and removal of fixation had little effect on the nystagmic response. In the peripheral group, removal of fixation enhanced the nystagmus and lessened the discrepancy between the two ears. Statistical procesing of the MRI showed that in the central group significant spatially coincident lesions occurred within the medial vestibular nucleus, lateral vestibular nucleus and proximal portion of the vestibular fascicle.
\end{abstract}

Caloric testing is an important part of neurootological assessment of the vestibular system, using a technique that has remained largely unchanged for the past 50 years. ${ }^{1}$ Abnormal responses take two basic forms: canal paresis and directional preponderance. In the former, with which we are primarily concerned here, the duration of the nystagmus evoked from the impaired ear by irrigation with water at $7^{\circ} \mathrm{C}$ above and below body temperature is reduced with respect to the normal side; normative data collected by Hallpike et $a l^{2}$ have shown that interaural differences of as little as $\mathbf{1 5}$ seconds may be significant. A paretic response indicates an ipsilateral lesion occurring at some point along the vestibular pathway, from the labyrinth to the vestibular nuclei situated in the floor of the fourth ventricle, and is thus of limited localising value. While it is most often due to a peripheral disturbance of vestibular function more central lesions can cause similar

Table 1 Clinical features of 20 patients with canal paresis.

\begin{tabular}{lll}
\hline & Central & Peripheral \\
& $n=10$ & $n=10$ \\
\hline Age: mean (range) & $31 \cdot 4(20-50)$ yrs. & $45 \cdot 6(24-64)$ yrs \\
Sex $(M / F)$ & $6 / 4$ & $5 / 5$ \\
Diagnosis & Definite MS & Peripheral Vestibular \\
Features & Brainstem Dysfunction & - \\
& 5 NOt & - \\
& $4>1$ cranial nerve palsy & - \\
& V -1 & \\
& VI -2 & \\
& VII -3 & \\
& mabnormal pursuit eye & - \\
& movements & \\
\hline
\end{tabular}

* Idiopathic or presumed hydrops/viral labyrinthitis † INO = Internuclear ophthalmoplegia

abnormalities. However, work in this area of "central" canal paresis has been hampered by lack of accurate anatomical localisation of brain-stem lesions. The development of MRI has improved both the definition of normal architecture within the brainstem ${ }^{3}$ and the detection of pathological lesions. ${ }^{4}$ This has allowed us to evaluate and correlate the occurrence of canal paresis with respect to lesions within the central vestibular pathways.

\section{Materials and methods}

Patients with central canal paresis were included in the study on the basis of the caloric finding, demonstrating a significant canal paresis (see below), and the presence of clinical evidence of brainstem disease. These patients had been referred to the MRC Hearing and Balance Clinic at Queen Square for the assessment of eye movement and/or balance disorders. For comparison an equal number of patients attending the same clinic with presumed peripheral vestibular dysfunction and observed canal paresis were assessed.

Ten patients were recruited to each group and their clinical features are shown in table 1 . All patients within the "central" group had established multiple sclerosis; by definition, the peripheral patients had no central neurological signs and were diagnosed as having a unilateral peripheral vestibular disorder, either idiopathic or suspected viral labyrinthitis ("vestibular neuronitis") or endolymphatic hydrops. There was a wide range in the duration of the vertiginous or brainstem symptoms in both groups of patients (one month to 20 years in the central group and two weeks to three years in the peripheral group) without significant differences between the two $(t=$ 1.63). All but one patient with central signs were less than 50 years of age; this lessened the risk of areas of age-related abnormal signal being misinterpreted on the MRI scan.

Caloric irrigation was performed by the authors according to Fitzgerald and Hallpike's method. ${ }^{1}$ The duration of the nystagmus was measured by direct observation, with and without visual fixation; the latter was achieved by donning Frenzel's glasses when the nystagmus was no longer apparent in the presence of fixation. Quantitative data were derived using the standard formula for canal paresis (CP):

CP $(\%)=\frac{(\text { left cold }+ \text { left hot })-(\text { right cold }+ \text { right hot })}{\text { (right cold }+ \text { right hot }+ \text { left cold }+ \text { left hot })} \times 100$

On the premise that an interaural difference 


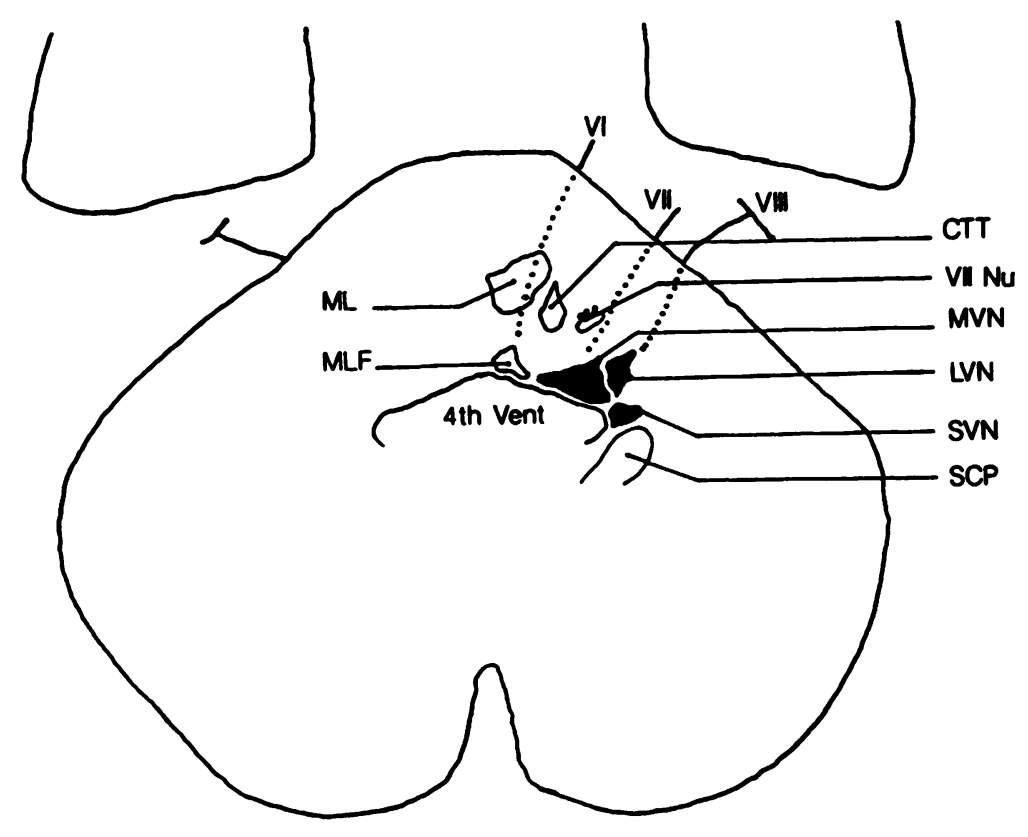

Figure 1 Schematic diagram showing the significant structures present at the level of the ponto-medullary junction. The vestibular nuclear complex is shown in solid black. The approximate trajectory of the VI, VII and VIII (vestibular) fascicles are indicated by dotted lines. SVN: superior vestibular nucleus; MVN: medial vestibular nucleus; LVN: lateral vestibular nucleus; MLF: medial longitudinal fasciculus; SCP: superior cerebellar peduncle, ML: medial lemniscus; CTT: central tegmental tract.

of at least 30 seconds was pathologically relevant (that is, 2 SD from a large normal population ${ }^{2}$ ), a canal paresis of greater than $8.3 \%$, using the formula above, was considered significant. For convenience we took $9 \%$ as our lower limit.

Each patient had standard T1 and T2-weighted MRI scans performed on a 0.5 Tesla Picker superconducting system. An image matrix of 128 by 256 was routinely employed, giving pixel dimensions of approximately $2.4 \mathrm{~mm}$ by $1.2 \mathrm{~mm}$. The most informative transverse brainstem image was at the level of the pontomedullary junction. Here the superior (SVN), medial (MVN) and lateral (LVN) vestibular nuclei can be localised to the most lateral aspect of the floor of the fourth ventricle (figure 1). Lesions occurring in more caudal slices of the brainstem were not resolved well enough in relation to brain stem anatomy for statistical analysis. Most patients had TR/TE $2000 / 60$ and TR/TI $1500 / 500$ sequences; lesions appearing as areas of increased signal (white) and low signal (black) respectively. Both sequences, and information from adja-

Table 2 Caloric findings in 20 patients with canal paresis of peripheral and central origin

\begin{tabular}{lll}
\hline & $\begin{array}{l}\text { Central } \\
n=10\end{array}$ & $\begin{array}{l}\text { Peripheral } \\
n=10\end{array}$ \\
\hline $\begin{array}{l}\text { Canal Paresis } \\
\text { (Mean and Range) }\end{array}$ & $31 \%(9-100 \%)$ & $61 \%(10 \cdot 7-100 \%)$ \\
$\begin{array}{l}\text { VORS Index } \\
\text { (Mean and Range) }\end{array}$ & $114 \%(100-128 \%)$ & \\
$\begin{array}{l}\text { Left nystagmus } \\
\text { Right nystagmus }\end{array}$ & $115 \%(100-126 \%)$ & $206 \%(130-280 \%)$ \\
Canal paretic side & $106 \%(100-136 \%)$ & $186 \%(130-259 \%)$ \\
Non paretic side & $128 \%(100-260 \%)$ & $196 \%(126-252 \%)$ \\
\hline
\end{tabular}

*VORS Index: vestibulo-ocular reflex suppression index, calculated as,

VORS Index $=\frac{\text { duration of nystagmus without fixation }}{\text { duration }} \times 100$ cent transverse brain slices, were used to determine the presence of lesions. The chosen brain slice from each patient was enlarged and reported blindly, that is, the radiologist did not know the caloric result or whether the patient belonged to the central or peripheral group. Unequivocal areas of abnormal signal were subsequently traced on acetate sheets overlying an anatomical template outlining the brainstem contours. Identified lesions were coded and analysed for their relevance to the noted canal paresis by a statistical method described fully in previous studies. ${ }^{56}$ Briefly, this tests whether a given number of lesions overlapping a defined area of brainstem within a group of patients, linked by a common clinical finding, have occurred by chance.

\section{Results}

\section{Caloric testing}

The results are summarised in table 2 . The canal paresis was generally of lower magnitude in patients with central lesions. Only three patients with central signs, compared with seven patients with peripheral vestibular dysfunction, had a canal paresis in which the responses from the affected ear were half the duration of those from the normal ear (that is, a canal paresis of $33 \%$ ).

Removal of fixation, by the use of Frenzel's spectacles, enhanced the nystagmus in eight patients with peripheral lesions; the discrepancy between the two ears becoming less than $9 \%$ in six. By contrast, none of the patients with central lesions showed enhancement on removal of fixation, reducing the degree of canal paresis to below $9 \%$ in only one. Vestibulo-ocular suppression (VORS) was further quantified with the formula:

$$
\text { VORS index }=\frac{\text { duration of nystagmus without optic fixation }}{\text { duration of nystagmus with optic fixation }}
$$

Use of this formula showed values close to 1 for patients with central lesions (absent VORS) and close to 2 for peripheral patients (preserved VORS) (table 2, right and left nystagmus shown separately). Interestingly, in the peripheral group, VORS indices obtained from the paretic ear were greater than those from the non paretic ear (paretic side $=1.96$ SD $0.49, \mathrm{n}$ $=5$; non paretic side $=1.42 \mathrm{SD} 0.09, \mathrm{n}=9 ; \mathrm{t}$ $=2.465, \mathrm{p}<0.05)$. Cases with total canal paresis, that is, nystagmus duration $=0$, or with spontaneous nystagmus in primary gaze, that is, nystagmus duration = infinity, were not included for these calculations.

Using the same criteria, seven patients with central lesions had a directional preponderance (DP) larger than $9 \%$. DP percentage being defined as:

$$
\text { DP }=\frac{(\text { left cold }+ \text { right hot })-(\text { left hot }+ \text { right cold })}{\text { (right cold }+ \text { right hot }+ \text { left cold }+ \text { left hot })} \times 100
$$

In three patients, as would be expected, this directional preponderance was to the side opposite the canal paresis but in four patients it was in the same direction. A directional preponderance was identified in four patients with peripheral lesions and it was in the opposite 
Table 3 Distribution of abnormal MRI signal at the ponto-medullary junction in 10 patients with central canal paresis (CP)

\begin{tabular}{|c|c|c|c|c|c|}
\hline & $\% C P$ & $M V N$ & $L V N$ & $S V N$ & Vest fasc \\
\hline 1 & 9.0 & - & - & - & - \\
\hline 2 & 35.8 & $++t$ & +++ & +++ & + \\
\hline 3 & 10.2 & ++ & - & - & - \\
\hline 4 & 9.4 & + & - & - & ++ \\
\hline 5 & 100.0 & + & - & - & - \\
\hline 6 & 100.0 & + & $++t$ & +++ & + \\
\hline 7 & 9.1 & ++ & + & + & - \\
\hline 8 & 9.9 & - & - & - & - \\
\hline 9 & 10.5 & +++ & +++ & - & +++ \\
\hline 10 & 13.5 & ++ & - & - & - \\
\hline
\end{tabular}

The + signs under a particular neural structure estimate their degree of intersection with an area of abnormal MRI signal $(+++$ indicates $100 \%)$. M, L and SVN: medial, lateral and superior vestibular nuclei; vest fasc: vestibular fascicle.

direction to the canal paresis in two of these.

\section{MRI findings}

All 10 patients with central canal paresis had an ipsilateral ponto-medullary lesion although in two cases the abnormal MRI signal did not intersect with the vestibular nuclei or fascicle (table 3). One patient, aged 53 years, with peripheral vestibular dysfunction had a pontomedullary lesion on MRI scanning but this was contralateral to her canal paresis and probably an incidental finding considering her age. All other scans in this group were normal at this level.

The results of the analysis to determine the anatomical significance of areas in which lesions overlapped, in patients with central canal paresis, is shown in figure 2 . The shaded and hatched areas in the grid matrix superimposed on the contours of the ponto-medullary junction represent the squares where levels of significance $<0.05$ were achieved. The anatomical structure contained within the shaded square $(\mathrm{p}<0.01)$ corresponded to a large portion of the medial vestibular nucleus and in the hatched squares $(p<0.03)$, in addition to

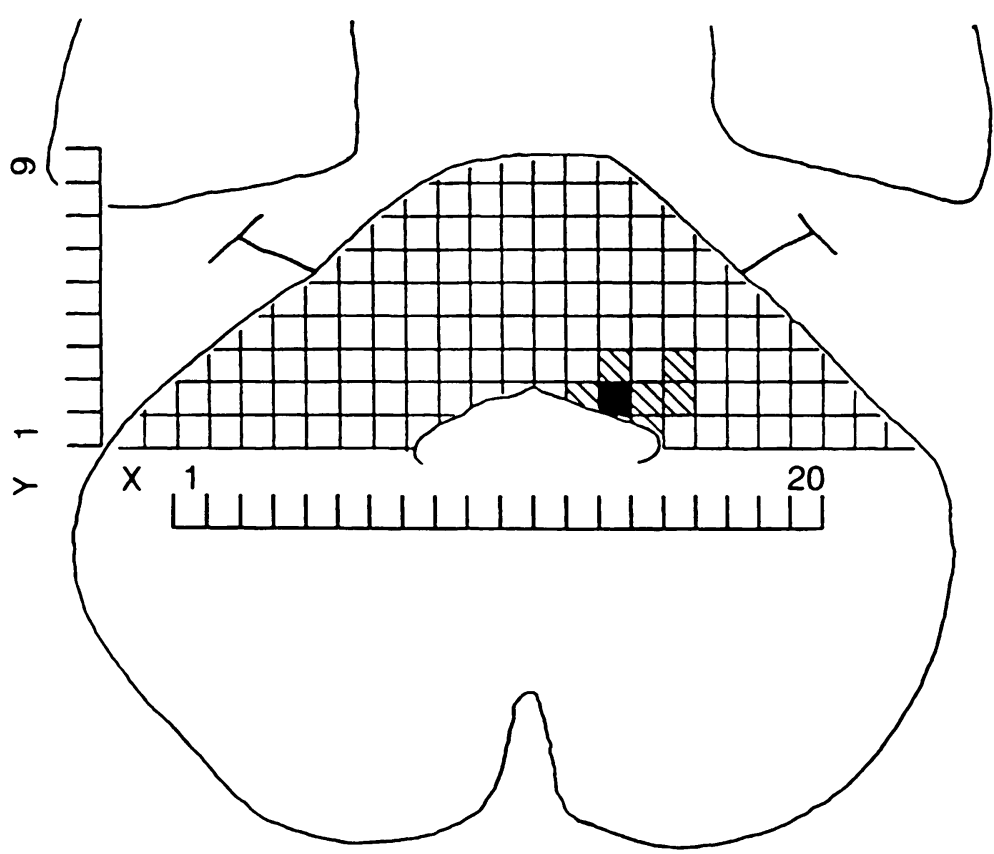

Figure 2 Drawing of the boundaries of the ponto-medullary junction with superimposed grid used to analyse the statistical significance of lesions at this level. The solid square signifies lesions attaining a significance of $p<0.01$, hatched squares $p<0.03$. the remainder of this nucleus, also included the lateral vestibular nucleus and most proximal portion of the vestibular fascicle. A comparison of figures 1 and 2 shows that the superior vestibular nucleus is relatively spared, as box X16-Y1 has no statistically significant overlapping.

\section{Discussion}

The vestibular nuclear complex consists of four separate nuclei each receiving afferent information from several different sources. The medial and superior vestibular nuclei receive information directly from the cristae of the semi-circular canals. ${ }^{7}$ In addition, the vestibular nuclei receive sensory input from the otolith organs, from proprioceptors, particularly from the neck, as well as visual information. The cerebellum is also a major source of afferent information, giving significant contributions to the superior, lateral and medial vestibular nuclei and overall has important inhibitory control upon vestibulo-oculomotor function. ${ }^{7}$

This anatomical arrangement explains two important observations in our study of caloric stimulation. The first is that the degree of canal paresis was in general of a lower magnitude in patients with central lesions when compared with those with abnormalities in the peripheral vestibular system. This agrees with previous experimental studies on primates showing that the greatest degree of canal paresis is produced by lesions in the vestibular nerve up to the level of the nerve root entry zone, the site at which the maximum number of afferent nerve fibres from the labyrinth could be damaged. ${ }^{8}$ Lesions in the nuclei related to canal afferents would have to be massive to achieve an equivalent deafferentation.

The second important point concerns the effect of removal of fixation upon the induced nystagmus. The removal of visual fixation produced enhancement of caloric responses in most of our patients with peripheral lesions but little significant change in those with central lesions. This finding is consistent with established observations on the relatively greater inhibitory effect of optic fixation on caloric nystagmus in peripheral compared with central vestibular lesions. ${ }^{9}$ Although our study is the first to show that lesions in the region of the vestibular nuclei do indeed cause a canal paresis that is unaffected by fixation, the fact that our clinical material comprises patients with demyelination makes it difficult to establish whether the association of canal paresis and reduced vestibulo-ocular reflex suppression arises in a single lesion or whether they are due to two or more coexisting lesions.

An unexpected finding in this study was that in the peripheral group the magnitude of nystagmus suppression by optic fixation was greater during irrigation of the paretic side than that of the non paretic side. The data from the study by Hood and Korres, ${ }^{9}$ using a similar caloric technique, was re-analysed and showed comparable findings to ours although the authors did not comment upon this point. The likely explanation for this finding is that the 
nystagmic response from the paretic side is more suppressed by vision simply because it is weaker, and therefore easier to suppress, than the one from the non paretic side. This is again supported by re-analysis of Hood and Korres data ${ }^{9}$ which shows that VORS indices from the paretic side $(2 \cdot 26)$ are higher than those from the normal population (1.85), giving the appearance of "hyper normal" visual suppression. In addition, the fact that VORS indices from the non paretic side were somewhat lower than normal, both in our study $(1.42)$ and in Hood and Korres data (1.65), might be due to the intense central inhibition exerted upon the vestibular system when there is a marked asymmetry between the two sides, such that any additional inhibition from the visual system is not apparent.

An important aim of this study was to identify what structures are involved in cases with central canal paresis. The data show that lesions affecting predominately the MVN, and to a lesser extent the $\mathrm{LVN}$ and proximal portion of the vestibular fascicle, appear to be the most important causes of "central" canal paresis, whereas the SVN is relatively spared. One limitation of this study has been the inability to identify components of the vestibular nuclear complex below the ponto-medullary junction. There is, however, no a priori reason to believe that more caudal structures are important in causing a canal paresis. Thus the rostral segment of the MVN is known to be a major terminus of afferent fibres from the semicircular canals which then projects onto the oculomotor system, whereas the central and caudal segments of this nucleus do not receive primary afferent vestibular fibres. ${ }^{1011}$

Although it is well established that the LVN is the source of origin of the vestibulo-spinal tracts, it is not clear whether this nucleus is involved in vestibulo-ocular function. Lesions produced by nerve root damage in primates caused severe degeneration of fibres in the ventral portion of LVN and suppressed ipsilateral horizontal caloric nystagmus. ${ }^{8}$ In cats a pathway linking LVN with abducens and medial rectus motor nuclei, via the ascending tract of Deiter's, has been described. ${ }^{12}$ The significant association between lesions in the LVN, the adjacent vestibular fascicle and canal paresis in our study might suggest a similar pathway exists in humans.

There is now good evidence that the major function of the SVN is mediating activity between the vertical semicircular canals and oculomotor nuclei controlling vertical and torsional eye movements. ${ }^{1314}$ In addition, Uemura and Cohen ${ }^{8}$ found that the horizontal component of caloric induced nystagmus was preserved after total SVN destruction. Our findings, showing a comparative lack of involvement of this nucleus in our patients with central canal paresis, are in agreement with this.

A final point that is crucial to the whole of this study is the accuracy with which the sites of various nuclei can be located. The position- ing of the head in the scanner, the variability of the orientation and the precise anatomy of the brainstem in different individuals and the relatively small size of the nuclei and fibre tracts of interest all combine to make accurate anatomical localisation hazardous. A small rotation of the brain relative to the scanner may move the centre of the slice of image by 1-2 $\mathrm{mm}$ at the level of the floor of the fourth ventricle relative to the anterior pons. Furthermore, conventional atlases of the brainstem usually depict transverse sections, while most of our transverse scans are in fact at an angle to this plane (that is, ca $10^{\circ}$ positive to the orbito-meatal line). We have attempted to obtain an average position of the lesions in this study superimposing them upon the assumed position of the vestibular muclei. Previously we have used a similar technique to study gaze palsies. $^{56}$ Comparison of the montages in these various studies will reveal small differences in the position of the nuclei at the level of the 8th nerve, differences that are easily obtained considering the $5 \mathrm{~mm}$ thickness of slices imaged and the anatomical factors described above. In spite of this we believe that this study is valid in demonstrating the approximate sites of lesions causing canal paresis. Clearer elucidation will require better visualisation of the individual nuclei and fibre tracts within each scan, which will overcome the problem of individual anatomical variation.

1 Fitzgerald G, Hallpike CS. Studies in human vestibular function. 1. Observations on the directional preponderence (Nystagmusbereitschaft) of caloric nystagmus resulting from cerebral lesions. Brain 1942;65:115-37.

2 Hallpike CS, Harrison MS, Slater E. Abnormalities of the caloric test results in certain varieties of mental disorder. Acta Otolaryngol 1951;39:151-9.

3 Flannigan BD, Bradley WG, Mazziotta JC, et al. Magnetic resonance imaging of the brain-stem: Normal structure and basic functional anatomy. Neuroradiol 1985;154: 375-83.

4 Ormerod IEC, Bronstein A, Rudge P, et al. Magnetic resonance imaging in clinically isolated lesions of the resonance imaging in clinically isolated lesions of the
brain stem. I Neurol Neurosurg Psychiatry 1986;49: brain stem.

5 Bronstein AM, Morris J, Du Boulay G, Gresty MA, Rudge P. Abnormalities of horizontal gaze. Clinical, oculographic and magnetic resonance imaging findings. I Abducens palsy. F Neurol Neurosurg Psychiatry 1990; 53:194-99.

6 Bronstein AM, Rudge P, Gresty MA, Du Boulay G, Morris J. Abnormalities of horizontal gaze. Clinical, oculographic and magnetic resonance imaging findings. II Gaze palsy and internuclear ophthalmoplegia. 7 Neurol Neurosurg Psychiatry 1990;53:200-7.

7 Wilson V, Melvill-Jones G. Mammalian vestibular physiology. New York : Plenum Press, 1979.

8 Uemura T, Cohen B. Effects of vestibular nuclear lesions on vestibulo-ocular reflexes and posture in monkeys. Acta

9 Hood JD, Korres S. Vestibular suppression in peripheral and central vestibular disorders. Brain 1979;102:785-804.

10 Gacek RR. The course and central terminations of first order neurons supplying vestibular end-organs in the cat. Acta Otolaryngol 1967;suppl 256:1-66.

11 Angaut P, Brodal A. The projection of the "vestibulocerebellum" onto the vestibular nuclei in the cat. Arch Ital Biol 1967;105:441-79.

12 Highstein SM, Baker R. Excitatory termination of abducens interneurons on medial rectus neurons; its relationship to the syndrome of internuclear ophthalmoplegia. $\mathcal{f}$ Neurophysiol 1978;41:1647-61.

13 McMasters RE, Weiss AH, Carpenter MB. Vestibular projections to the nuclei of the extraocular muscles. Degeneration resulting from discrete partial lesions of the vestibular nuclei in the monkey. Amer f Anat 1966; 118:163-94.

14 Tokomasu K, Goto K, Cohen B. Eye movements from vestibular nuclei stimulation in monkeys. Ann Otol 1969; 78:1105-19. 